\title{
Application Study of Fuzzy Reasoning Method for Domain Ontology
}

\author{
Hong Wang, Jian Li, Qingqing Zhang and Weiwei Cai \\ College of Computer Science and Technology, Civil Aviation University of China, \\ Tianjn, 300300, China \\ hwang@cauc.edu.cn, ljian7115@sina.com,j_wang@cauc.edu.cn
}

\begin{abstract}
For the representation and reasoning of fuzzy knowledge for domain ontology, the paper presents the reasoning method based on fuzzy ontology. On a basis of the study of civil aviation emergency domain ontology, it gives fuzzy extension model of domain ontology based on fuzzy description logic from the perspectives of fuzzy modifiers and fuzzy concrete domains, and designs fuzzy rules by introducing weight concept and gives the representation and construction process of fuzzy rules on a basis of $f$-SWRL in connection with the inference application of situation analysis for civil aviation events. The experimental results show that the fuzzy extension method of domain ontology can make up for the issue that the existing domain ontology is inadequate in terms of fuzzy knowledge representation, and provide a good methodological support for making domain ontology perfect and inference application with the reasoning implementation based on fuzzy rules.
\end{abstract}

Keywords: fuzzy rules, civil aviation events, situation analysis, reasoning

\section{Introduction}

Domain ontology is a description of the specific areas concepts and their interrelations, which is based on clear description logic[1], so deficiencies exist in representation and reasoning for fuzzy knowledge. In terms of constructing fuzzy ontology, Zhai Jun, who introduced the fuzzy concepts and relationships into common ontology model, which researched fuzzy ontology model based on linguistic variables [2]. Fuzzy domain ontology model was built on the basis of this model, used in university research [3], graduates [4], intelligent transportation [5], e-commerce [6] and other areas. Li hui-lin, Ma di and other proposed construction method of fuzzy ontology based on FFCA [7-8], which is a semi-automatic construction method of fuzzy ontology, but this method is still inadequate in dealing with Chinese data source. Femando Bobillo, Umberto Straccia do research on fuzzy $O W L 2$ in application of $O W L$ ontology, using OWL2 annotation attributes to define fuzzy concepts, fuzzy relations and fuzzy membership functions, achieving good expression of fuzzy ontology [9-11]. Lee proposed a fuzzy ontology model that mainly includes the domain layer, event level, category level and class level, by integrating membership function into the concepts, and using fuzzy relations to represent the linkages of concepts [12-14]. In the term of ontology inference, Sun Peng conducted a related study on ontology inference based on description logic for the Semantic Web [15], proposing an improved algorithm ERXM. Tang Xiaobo and others do the relevant research on semantic inference based on ontology and rules [16], through the establishment of rules and logical reasoning, deduced a new ontology on the basis of the original ontology achieving the implicit relationship of ontology members between semantic reasoning, improving the ontology knowledge, but deficiencies exist in the fuzzy knowledge reasoning. 
The construction of civil aviation emergency domain ontology, achieves a good expression sharing and reuse of domain knowledge, while on the basis of domain ontology, the generation of the initial rescue plan has achieved with semantic search and reasoning mechanisms and the service platform for emergency management has been established [17-18]. But in the representation of fuzzy knowledge had not yet been involved, it is difficult to support uncertain information describing to emergency response process and reasoning applied research and other problems. This paper introduces fuzzy theory and based on the civil aviation emergency domain ontology CAEDO, for its fuzzy extension [19], and builds the fuzzy rules, combined with the practical application of situation analysis of civil aviation events, and realizes inference based on fuzzy ontology. According to the past experience knowledge and comprehensive analysis for the situational factors and decision indicators of civil aviation events, fuzzy rules of situation analysis for civil aviation events are designed and constructed. The implementation of inference based on fuzzy ontology and rules for situation analysis provides decision support for civil aviation emergency rescue.

\section{Extension Process of Domain Ontology Based on Fuzzy Description Logic}

In the actual application process, fuzziness of things is reflected in the following two aspects: (1) vagueness that certain things belong to a concept or relationship with different degrees caused by the introduction of natural language; (2) uncertainty for range of things because of modification of fuzzy modifiers.

For these two types of fuzzy information, fuzzy modifiers and fuzzy concrete domains of fuzzy description logic have been introduced to achieve the fuzzy extension for the concepts and relations in domain ontology. The extended model of domain ontology based on fuzzy description logic is designed as shown in Figure 2.1, and the specific implementation is shown in [19].

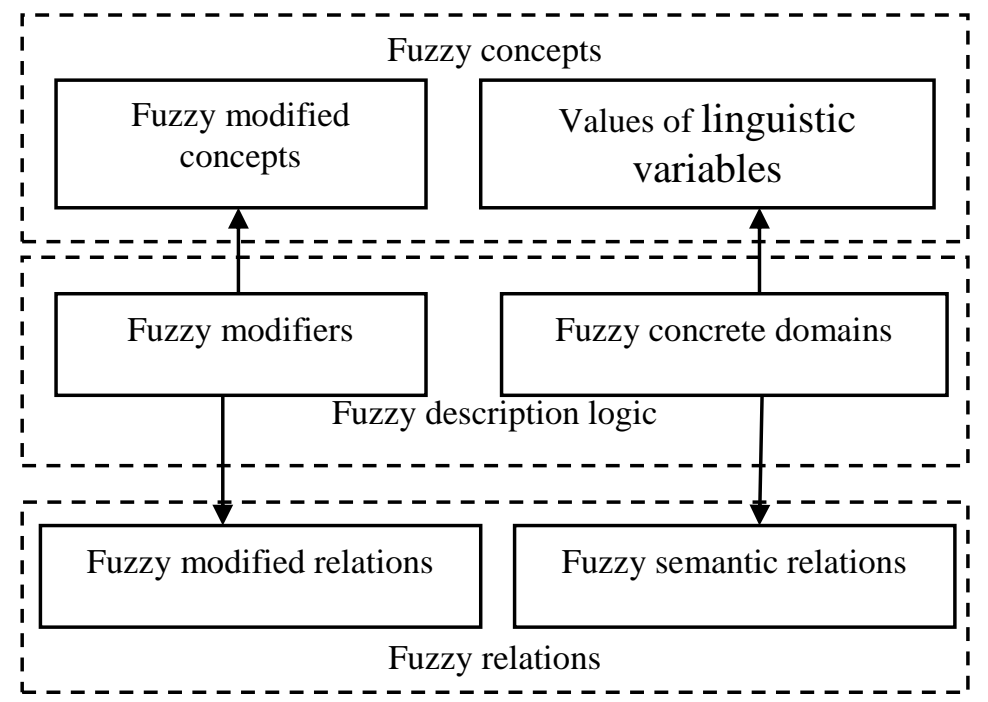

Figure 2.1. The Extended Model of Domain Ontology Based on Fuzzy Description Logic 


\section{Inference Based on Fuzzy Ontology and Rules}

In the fuzzy inference process, fuzzy rules are essential, which are constructed according to the specific application requirements and describe more complex semantic relationships. And services are provided for specific applications by reasoning based on domain ontology extended and fuzzy rules.

\subsection{Inference Process Based on Fuzzy Ontology and Rules}

Jena, API for RDF and $O W L$ developed by HP Labs, is the application development package with comprehensive content. In the inference process based on ontology and rules, ontology is parsed to collection of asserted triples with Jena, and then the inferred triples are obtained by inference engine reasoning. The final inferred triples are obtained through the rules inference engine by combing domain rules and inferred triple and reasoning. The inference process based on fuzzy ontology and rules is shown in Figure 3.1 .

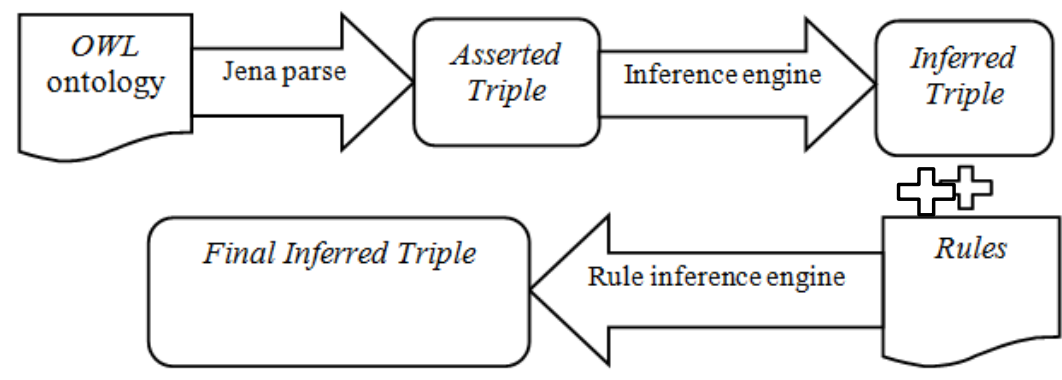

Figure 3.1. The Inference Process Based on Fuzzy Ontology and Rules

\subsection{The Design and Representation of Fuzzy Rules}

Since the information is often inaccurate in the specific practical application, the paper introduces fuzzy concept to represent fuzzy rules, obtaining the information to match facts in the maximum degree. Inaccurate information with fuzzy representation is processed through membership calculation.

Fuzzy production rules are represented as: $(P) / T \rightarrow Q$. The value of $P$ is fuzzy concept and $T$ refer to the truth degree of premise. Note that: the division of fuzzy sets generally is based on empirical knowledge and domain knowledge so as to make distribution reasonable and be able to better cover the domain of discourse.

Typically, rules contain a plurality of preconditions, whose importance to the conclusion is set to the same. In order to improve the ability of knowledge expression of the rules and reduce the loss of useful information, and improve the inference accuracy, the paper introduces the concept of weights, to distinguish different influence degrees of the prerequisites for the conclusion. For example, for a certain type of emergency, situation of event has many manifestations, and different situations correspond to different emergency actions. In other words, the importance of different situation for the same event is different. Generally, the fuzzy weights degree is determined by negotiating among the experts.

Experts in specific areas give fuzzy weights to certain preconditions of rules, and then the rule base is established containing fuzzy characteristics. The production representation of fuzzy rules based on weights is defined as follows:

$$
\left(w_{i 1} * P_{i 1}\right) / T_{i 1},\left(w_{i 2} * P_{i 2}\right) / T_{i 2}, \ldots,\left(w_{i n} * P_{i n}\right) / T_{i n} \rightarrow Q_{i}
$$


Where $w_{i j} \geq 0$, and $\sum_{\mathrm{j}=1}^{\mathrm{n}} w_{i j}=1$. $w_{i j}$, weight, refer to the importance degree of preconditions $P_{i j}$ to conclusion. $T_{i j}$ refer to the truth of preconditions $P_{i j}$, and the value of $T_{i j}$ is calculated as follows: if $u$ refer to an element, the value of $T_{i j}$ is the membership degree of $u$ to $P_{i j}$; And if $u$ refer to fuzzy set, the value of $T_{i j}$ is the similarity degree of $u$ to $P_{i j}$, namely, $M\left(P_{i j}, u\right)$ refer to the maximum membership degree among the two sets.

$$
T_{i j}= \begin{cases}\mu(u) & u \text { refer to an element } \\ M\left(P_{i j}, u\right) & u \text { refer to fuzzy set }\end{cases}
$$

For instance, $P$ represent that the number of deaths is many, and if the value of $u$ is 35 , the membership degree of $u$ belonging to many is 0.8 , in other words, the value of $T$ is 0.8 ; if the value of $u$ is many, the value of $T$ is 1 .

The truth of preconditions $T_{i}$ is calculated according to the following formula:

$$
T_{i}=\sum_{j=1}^{n} w\left(P_{i j}\right) \cdot T\left(P_{i j}\right)
$$

Note that: the value range of $P_{i j}$ is the value of linguistic variable.

Fuzzy modified concepts and language variable can be transformed each other. For example, when $P_{i j}$ represent that the number of deaths is many, the membership degree of about 30 belonging to many is obtained by calculating their common point, namely two intersection of the curve.

\subsection{The Implementation of Fuzzy Inference Rules}

To analyze accurately the semantic relations of domain knowledge, and to design appropriate rules and represent them formally with description language, which determine the completion of rule-based ontology reasoning and the completion effect. The main steps for constructing rules are shown as follows.

(1)To determine the condition attributes and decision attributes, namely attribute set of premise and conclusion for rules, respectively.

(2)To determine the range set of condition attributes and decision attributes.

(3)Attribute Reduction.

Assuming that rule is $P \rightarrow Q$, if and only if the rule satisfies $(P-\{a\}) \rightarrow Q$, attribute

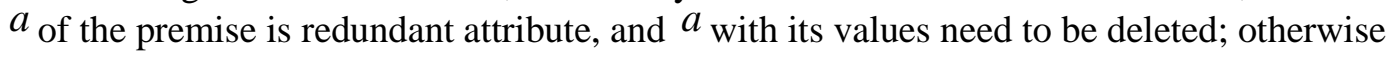
attribute is necessary, which need to retain.

(4) To define weight for each attribute of the after reduction. The weight is generally identified by experts in the field, and to ensure that the weight sum of each item in premise is 1 .

(5)To assemble rules according to the value of attribute, namely the rules that we want to build. 


\section{Application of Inference for Situation Analysis of Civil Aviation Events}

\subsection{The Situation Analysis of Civil Aviation Events}

The emergency management regulations for civil aviation of China provides that events of civil aviation refer to sudden emergencies threating or hazarding to civil aviation activities, which has caused or may cause significant casualties, property damage or other serious harm to society, and need take emergency measures to deal with, as well as civil aviation activities to avoid or deal with other types of public emergencies.

Timely and effective emergency disposal and making better use of the collected information about events and emergency resources is on a basis of conducting situation analysis. Civil aviation events are complex, serious harm, disposal difficult, however the situation information is local predictable due to its information gathering. On a basis of the study of civil aviation events, a comprehensive analysis and prediction to development trends and influence scope is conducted by combining state information on-site with expert experience and historical data, so as to determine resource requirements for emergency rescue and mobility program, emergency rescue services, providing decision support for decision makers.

The influence factors of situation information are complex, information about the event and the factors that may affect the development of the event need to be obtained as comprehensive as possible before making trend forecasts. Taking the characteristics of civil aviation events into account, the paper will summarizes and analyses the influencing factors from the following three aspects.

(1) Event basic information

Including the location of the event, event type, aircraft type, aircraft extent of the damage, casualties and so on.

(2) Environmental information

Environmental factors mainly refer to weather factors, this paper weighs environmental factors from the slippery degree of the road and visibility.

(3) Emergency disposal information

Including emergency response time, and the number of emergency resources.

Civil aviation events, in accordance with factors, such as nature, severity, controllability, and influence scope, are generally divided into four grades: particularlySignificant (I grade), significant(II grade), comparativelySignificant(III grade), generallySignificant (IV grade). Therefore, index of situation analysis of the event is mainly considered and summarized as follows:

(1) Severity analysis of event: mainly including event level, casualties, the extent of damage to the aircraft, aircraft type, locations of event.

(2) Disposal difficulty of rescue: mainly including the protection level of emergency resources, emergency capacity of personnel, emergency response time, slippery degree of road, visibility.

(3)Degree of influence expanded: environmental factors, occurred time of event, the occurred location, influence of occurred location.

The fuzzy concepts based on linguistic variables are summarized in Table 4.1, on a basis of analyzing the relational factors in emergency procedure, and existing domain ontology and domain characteristics. Considering the fuzzy division not too thick or too thin, the process state with too thick division is inaccurate or cannot be characterized, and the computation and the reasoning difficulty are greatly increased if the division is too thin. Thus, the value is generally divided into three or four grades. 
Table 4.1. Definition of Concepts Based on Linguistic Variables

\begin{tabular}{|c|c|c|}
\hline Category & linguistic variables & Values of linguistic variables \\
\hline $\begin{array}{l}\text { Event basic } \\
\text { information }\end{array}$ & $\begin{array}{l}\text { Event Level } \\
\text { Damage extent } \\
\text { Casualties } \\
\text { Aircraft type } \\
\text { Influence scope }\end{array}$ & $\begin{array}{c}\text { particularlySignificant, significant, } \\
\text { comparativelySignificant, } \\
\text { generallySignificant } \\
\text { severelyDamaged,generallyDameged, } \\
\text { slightlyDamaged } \\
\text { many,general,few } \\
\text { large scale,Mediumscale,Small-scale } \\
\text { wide,general,small }\end{array}$ \\
\hline $\begin{array}{l}\text { Environmental } \\
\text { information }\end{array}$ & $\begin{array}{c}\text { Visibility } \\
\text { Slippery degree of road }\end{array}$ & $\begin{array}{c}\text { (fine),(mist, heavy rain),(fog),(heavy fog) } \\
\text { (fine,light rain),(rain,light snow),(heavy } \\
\text { rain,snow),(rainstorm,heavy snow) }\end{array}$ \\
\hline $\begin{array}{l}\text { Emergency } \\
\text { disposal } \\
\text { information }\end{array}$ & $\begin{array}{l}\text { Emergency response time } \\
\text { Protection level of resources } \\
\text { Disposal difficulty of rescue } \\
\text { Emergency disposal speed }\end{array}$ & $\begin{array}{l}\text { fast ,moderate,slow } \\
\text { good,general,poor } \\
\text { difficulty,moderate,easy } \\
\text { fast,moderate,slow }\end{array}$ \\
\hline
\end{tabular}

According to the procedure described in Section 3.3, the $f$-SWRL rules for situation analysis of events are described in Table 4.2.

\subsection{The Implementation Process of Fuzzy Inference}

The inference realization use Jena and FuzzyDL [20] to superimpose reasoning, which is based on fuzzy ontology and rules. Reasoning model is created by associating ontology data and inference engine with ModelFactory, and then by querying, the original data and data obtained through other mechanisms or rules inference are received. The core codes are shown as follows.

(1)Model establishment and data loading

The paper chooses semantic framework Jena [21-22]. Jena package has ontology subsystem, which can read ontology and deal with ontology with ontology model (OntModel). When using Jena to process ontology, to create an empty ontology model first, and then to write the contents of ontology to this model. Jena uses the method ModelFactory of package OntModel to create ontology model, and the method read of OntModel to read the $O W L$ file from disk. The following code shows how to create a memory-based model, and to realize the process of data loading.

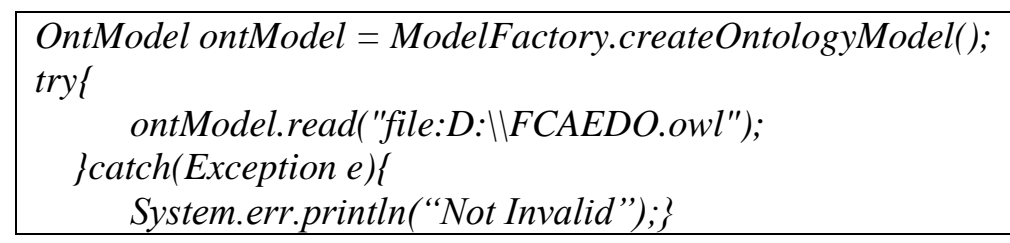

(2) To create instance data for concepts of civil aviation emergency domain ontology according to basic emergency status of events. 


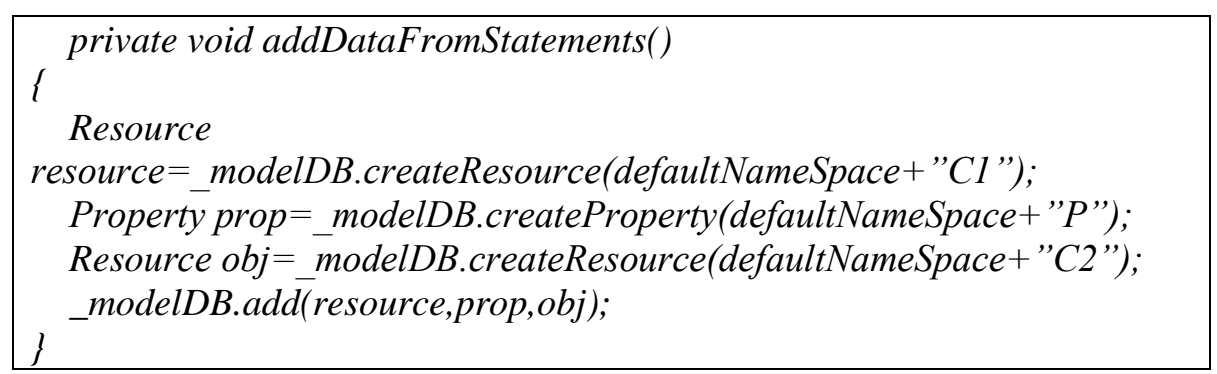

Where $P$ represent object attributes, such as belongToEventType, has Casualty. (3) To load rules file, and use the appropriate inference engine to reason.

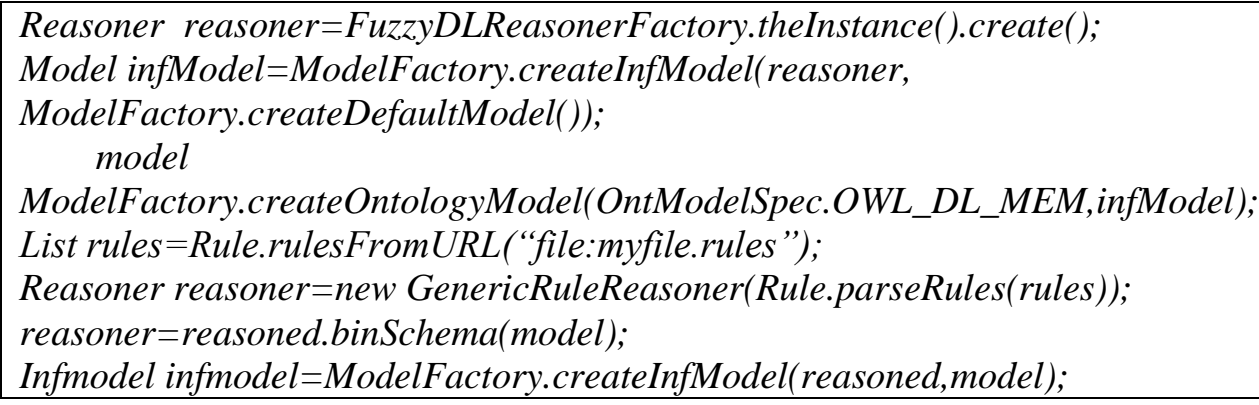

Table 4.2. The $f$-SWRL Rules for Situation Analysis of Events

\begin{tabular}{|c|c|c|c|}
\hline $\begin{array}{c}\text { Rules } \\
\text { Category }\end{array}$ & $f-S W R L$ & $\begin{array}{c}\text { Rule } \\
\text { explanation }\end{array}$ & $\begin{array}{l}\text { The } \\
\text { number } \\
\text { of rules }\end{array}$ \\
\hline $\begin{array}{l}\text { RULE-1: } \\
\text { Severity } \\
\text { analysis of } \\
\text { event }\end{array}$ & $\begin{array}{c}\text { Emergency }(? x) \wedge \text { belongToEventType }(? x, ? y) \wedge \\
w 1 * \text { damagedCondition }(? x, ? z) \wedge \\
w 2 * \text { hasCasualty }(? x, ? c) \rightarrow \text { eventLevel }(? x, ? p)\end{array}$ & $\begin{array}{l}\text { ?x event } \\
\text { name; } \\
\text { ?y event } \\
\text { type }\end{array}$ & 60 \\
\hline $\begin{array}{l}\text { RULE-2: } \\
\text { Disposal } \\
\text { difficulty } \\
\text { of rescue }\end{array}$ & 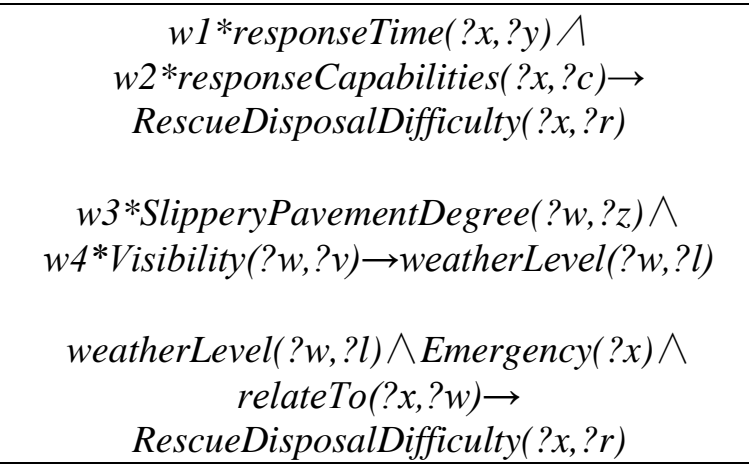 & $\begin{array}{l}\text { ?z slippery } \\
\quad \text { degree of } \\
\quad \text { road; } \\
\text { ?v visibility; } \\
\text { ?r Disposal } \\
\text { difficulty of } \\
\text { rescue. }\end{array}$ & 18 \\
\hline $\begin{array}{l}\text { RULE-3: } \\
\text { Degree of } \\
\text { influence } \\
\text { expanded }\end{array}$ & $\begin{array}{c}\text { Emergency }(? x) \wedge w 1 * \text { occuredTime }(? x, ? y) \wedge \\
w 2 * \text { occurSite }(? x, ? z) \rightarrow \\
\text { eventsExpansionLevel }(? x, ? l)\end{array}$ & $\begin{array}{l}\text { ?l Degree of } \\
\text { influence } \\
\text { expanded }\end{array}$ & 12 \\
\hline
\end{tabular}

\subsection{The Application Effect Analysis of Inference}

The basic principles of organization implementation rescue for civil aviation airport are centralized management, unified command, rapid response and rescue efficient. After the event occurring, the relevant departments of airport analyze comprehensively information 
of situation, such as the influence scope, the effect way, the duration and degree of harm, and predict derived disasters timely and adjust emergency rescue plan in order to assist emergency personnel decision-making and prevent events that have occurred causing more serious disaster, and control, reduce and eliminate harm caused by unexpected events.

Emergency rescue decision, as an important part of the management process, is a typical non-programmed decision problem that requires a series of processes and operations, such as data analysis, the model processing, knowledge reasoning, to support.

Important application of inference based on ontology and rules in civil aviation Emergency Management is emergency rescue aid decision, which involves optimization for decision-making information and contains complicated process. Maker enter the basic situation of event into system, and the better decision-making information is obtained through reasoning mechanism, which is based on fuzzy ontology and rules.

For instance, the information of event $E$ is described as follows:

Event address: Eastern 1st runway B2 slippery of some Airport

Event time: December 5, 2014, T16: 26: 13

Event description: landing gear severely damaged, aircraft generally damaged

The number of deaths: about 20

The number of injured: about 30

Environmental status: light snow

Through Jena resolution, ontology instance data, which is the data entry of engine, are generated by combining emergency status information data with the concept of ontology, while ontology and fuzzy rules is the rule entry. The inference result is received through inference engine operation. The process of example is shown as follows.

The ontology instances of event status information are generated through Jena parse:

(E,hasAddress, Eastern 1st runway B2 slippery of some Airport) ;

(E, hasDate, December 5, 2014 T16: 26: 13) ;

(E, hasCasualty, about 20).

The inference based on ontology concept is occurred and inference engine selected here is FuzzyDL: E, representing Emergency, can infer objects Aircraft, eventLevel, according to object attribute relateTo, hasEventLevel respectively; and infer Casualty based on data attribute hasCasualty. Aircraft can infer object damageExtent, according to object attribute hasDamageExtent. Namely:
(E, hasType, Emergency) ;
(E, relateTo, Aircraft) ;
(EventType, relateTo, address) ;
(E, hasEventLevel, eventLevel);
(E, hasCasualty, Casualty) ;

(Aircraft, hasDamageExtent, damageExtent).

Owing to the fact that the address of $E$ is inside the airport and $E$ is related to Aircraft, according to the inference rule,

Emergency(E) 1 hasAddress(E,inside of Airport) 1 relateTo(E, Aircraft)

$\rightarrow$ EventType(E, Aircraft_venue_crashed)

the conclusion is obtained:

(E, EventType, Aircraft_venue_crashed) ;

According to the rule,

Emergency $(E) \wedge$ belongToEventType(E, Aircraft_venue_crashed $) \rightarrow$ 
ToActiveRescuingPlan(E, Aircraft_VenueRescueProcedures)

the result is inferred:

(E, ToActiveRescuingPlan, Aircraft_VenueRescueProcedures) ;

Similarly, the deterministic inference result, such as rescue units, rescue headquarters, is obtained.

According to the fuzzy rule

Emergency $(? x) \wedge$ belongToEventType $(? x, ? y) \wedge w 1 *$ damagedCondition $(? x, ? z) \wedge$

$w 2 *$ has Casualty $(? x, ? c) \rightarrow$ eventLevel $(? x, ? p)$

The membership degrees of about 20 belonging to many, general, few are 0.3, 0.6, 0 , and according to the rule, the conclusion is:

(E, hasEventLevel, comparativelySignificant) ;

namely, the event level of $E$ is comparativelySignificant.

The slippery degree of road is Igrade, because the weather is snow; the response time of emergency rescue departments is $5 \mathrm{~min}$, and the membership degree of $5 \mathrm{~min}$ belonging to fast is maximum. Thus,

(E, responseTime, $5 \mathrm{~min})$;

(E. relatedTo, wetherLevel) ;

(SlipperyPavementDegree, hasValue, 1grade).

The disposal difficulty of event is medium according to the determination rule of disposal difficulty.

The occurrence time of event is afternoon and the address is inside of airport, combing with the rule of disposal difficulty, and according to the fuzzy rule

Emergency $(? x) \wedge w 1 *$ occuredTime $(? x, ? y) \wedge w 2 *$ occurSite $(? x, ? z) \rightarrow$

eventsExpansionLevel $(? x, ? l)$

Emergency $(? x) \wedge$ RescueDisposalDifficulty $(? x, ? r) \rightarrow$ eventsExpansionLevel $(? x, ? l)$

the expansion level of event is small.

In summary, the fuzzy domain ontology has advantages in semantic information description and processing with its good information organization and semantic inference ability. Inference based on fuzzy ontology and rules can not only obtain certain knowledge, but get uncertain knowledge. The experimental results show that the fuzzy domain ontology can represent fuzzy information better and reason combing with fuzzy rules, and the decision-making knowledge can be generated accurately to meet the real needs of decision-makers through the situation analysis based on fuzzy ontology and rules, according to natural language, to better assist emergency rescue.

\section{Conclusions}

On a basis of existing domain ontology, the paper has given the extension process of domain ontology, designs fuzzy rules by introducing weight concept and gives the representation and construction process of fuzzy rules on a basis of $f$-SWRL in connection with the inference application of situation analysis for civil aviation events, and introduced fuzzy inference based on fuzzy ontology and rules, providing a good methodological support for making domain ontology perfect and inference application. Next, a study for automation building of ontology and optimization of fuzzy rules to make them more reasonable and standard will be conducted.

\section{Acknowledgements}

This work is supported by the National Natural Science Foundation of China under Grant No. 61079007, the National Nature Science Youth Foundation of China under Grant No. 61301245, and the Fundamental Research Funds for the Central Universities of Civil Aviation University of China under Grant No. 3122014C016. 


\section{References}

[1] S. Rudi, R. Benjamins and D. Fensel, "Knowledge Engineering: Principles and Methods", Data and Knowledge Engineering, vol. 25, no. 1-2, (1998), pp. 161-197.

[2] Z. Jun, "Representation and construction for fuzzy ontology of linguistic variables", Computer \& Digital Engineering, no. 9, (2009), pp. 113-115.

[3] J. Zhai, Y. Liang, J. Jiang and Y. Yu, "Ontology-Based Information Retrieval for University Scientific Research Management", 4th International Conference on Wireless Communications, Networking and Mobile Computing(WiCOM '08), (2008), pp. 1-4.

[4] X. Hu, Z. Wang, J. Zhai and J. Wang, "A Mobile Service Application Platform with Chinese Short Message Based on Fuzzy Ontology for Employment of University Graduates", Eighth International Conference on Mobile Business (ICMB 2009), (2009), pp. 164-169.

[5] J. Zhai, Y. Cao and Y. Chen, "Semantic Information Retrieval Based on Fuzzy Ontology for Intelligent Transportation Systems", IEEE International Conference on Systems, Man and Cybernetics (SMC 2008), (2008), pp. 2321-2326.

[6] J. Zhai, L. Shen, Y. Liang and J. Jiang, “Application of Fuzzy Ontology to Information Retrieval for Electronic Commerce", 2008 International Symposium on Electronic Commerce and Security, (2008), pp. 221-225.

[7] L. H. Lin, "Research on semantic web fuzzy ontology construction based on FFCA", Dalian: Dalian maritime university, (2011).

[8] M. Di, "FFCA based fuzzy ontology learning method research", Dalian: Dalian maritime university, (2013).

[9] F. Bobillo and U. Straeeia, “An OWL Ontology for Fuzzy OWL2”, SPringer-Verlag Berlin Heidelberg, (2009), pp. 151-160.

[10] F. Bobillo and U. Straccia, "Representing Fuzzy Ontology in OWL 2", 2010 IEEE World Congress on Computational Intelligence (WCCI 2010), (2010).

[11] F. Bobillo and U. Straccia, "Fuzzy Ontology Representation using OWL 2", International Journal of Approximate Reasoning, (2011), pp. 1-36.

[12] C. S. Lee, Y. J. Chen and Z. W. Jian, "Ontology-based fuzzy event extraction agent for Chinese e-news summarization", Expert Systems with Applications, vol. 25, no. 3, (2003), pp. 431-447.

[13] C. S. Lee, Z. W. Jian and S. M. Guo, "Weighted Fuzzy Ontology for Chinese e-News Sununarization", BudaPest, Hungary, (2004), pp. 25-29.

[14] C. S. Lee, Z. W. Jian and L. K. Huang, "A fuzzy ontology and its application to news summarization", IEEE Transactions on Systems, Man and Cybernetics, Part B, vol. 35, no. 5, (2005), pp. 859-880.

[15] P. Sun, "Research on ontology reasoning based on description logic in semantic web", Jilin University, vol. 5, (2009).

[16] T. Xiaobo and J. Zhongming, "Research on semantic reasoning with ontology and rules", Journal of the china society for scientific and technical information, vol. 30, no. 7, (2011), pp. 695-703.

[17] H. Wang, Z. Pan and W. Zhou, "The Design and Implementation of Information System in Civil Airport Emergency Management Based on Semantic Web", 2013 25th Chinese Control and Decision Conference(CCDC), (2013).

[18] Z. W. Tao, "Research on Constructing Semantic Model of Emergency Decision-making Plan for Civil Aviation", Application Research of Computers, vol. 30, no. 1, (2013), pp. 195-198.

[19] H. Wang, J. Li and J. Wang, "Research on Fuzzy Extension of Domain Ontology for Emergency Management of Civil Aviation”, Journal of Information \& Computational Science, vol. 11, no. 16, (2014), pp. 6019-6028.

[20] F. Bobillo and U. Straccia, "FuzzyDL: An expressive fuzzy description logic reasoned", In Proceedings of International Conference on Fuzzy Systems, (2008), pp. 923-930.

[21] D. Reynolds, "Jena 2 Inference Support[EB/OL]", (2010).

[22] J. Carroll, I. Dickinson and C. Dollin, "Jena: implementing the semantic web recommendation", In Proceedings of the $13^{\text {th }}$ International World Wide Web Conference on Alternate Track Paper \& Posters. (2004), pp. 74-83. 


\section{Authors}

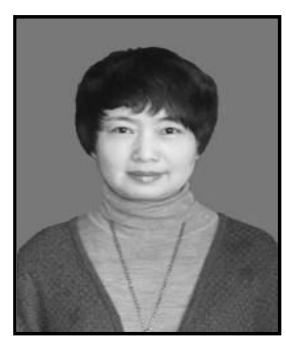

Hong Wang, born in 1963, professor, her research interests include ontology technology, data mining, and intelligent information processing.

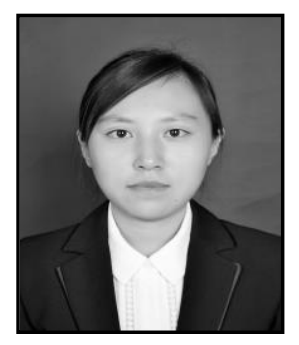

Jian Li, born in 1988, MS candidate, her research interests include ontology and civil aviation information system. 
International Journal of Database Theory and Application Vol.9, No.8 (2016) 